\title{
Strain partitioning between nerves and axons: estimating axonal strain using sodium channel staining in intact peripheral nerves
}

\author{
Fabio Bianchi ${ }^{1}$, Ruby Sedgwick ${ }^{2}$, Hua Ye $^{1}$ and Mark S. Thompson ${ }^{1 *}$ \\ ${ }^{1}$ Institute of Biomedical Engineering, Dept. of Engineering Science, University of Oxford, \\ Oxford OX3 7DQ, UK \\ 2 Department of Engineering Science, University of Oxford OX1 3PJ, UK \\ E-mail: hua.ye at eng.ox.ac.uk
}

\footnotetext{
* Corresponding Author Prof. Mark S. Thompson, Institute of Biomedical Engineering, Botnar Research Centre, Headington, Oxford OX3 7LD, UK. Tel: +44(0)1865 737845
} 

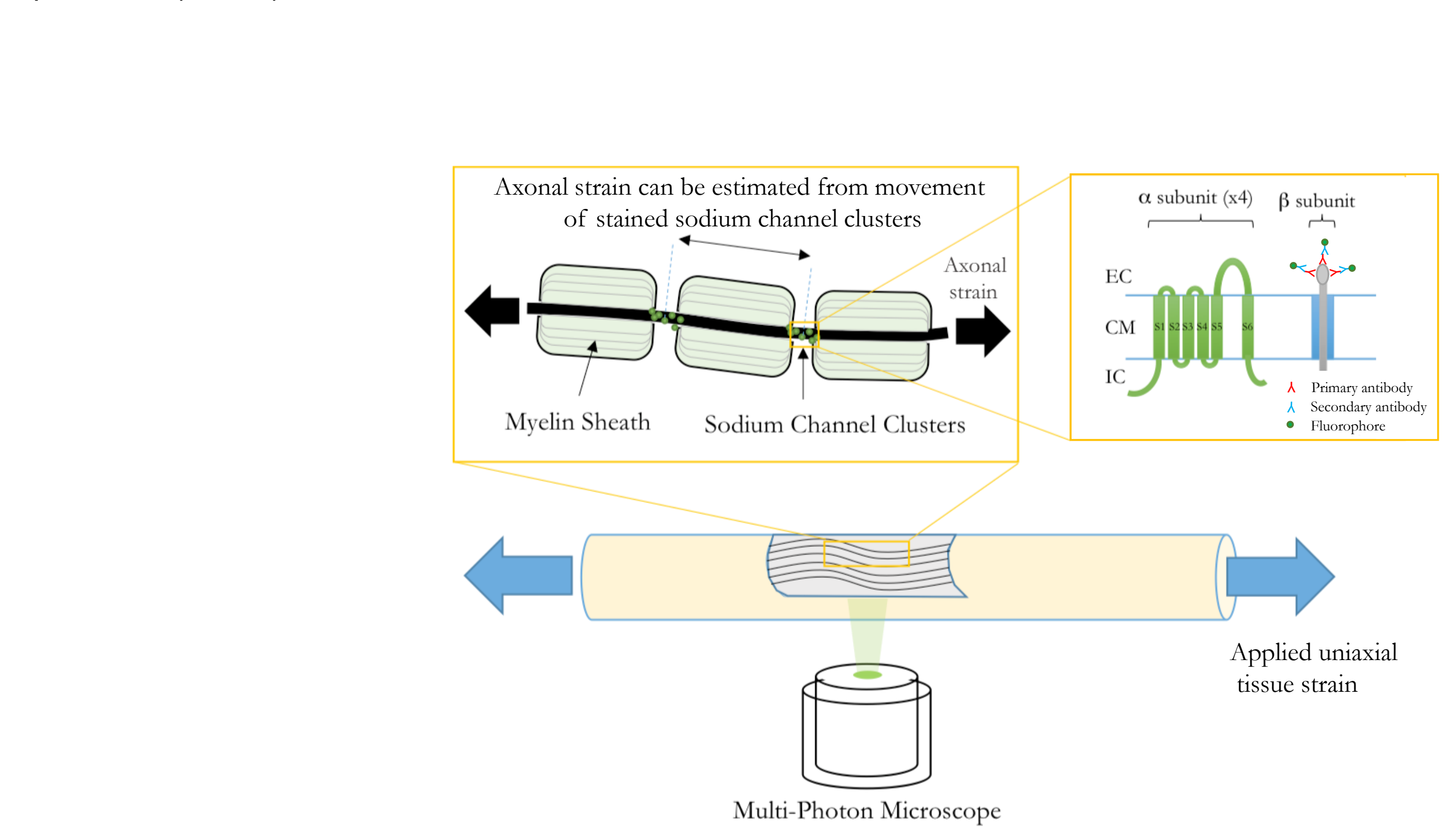

Applied uniaxial tissue strain

Multi-Photon Microscope 
Research Highlights for: Strain partitioning between nerves and axons: estimating axonal strain using sodium channel staining in intact peripheral nerves

- Partitioning of axial strain from whole nerve to axonal fibres is not known

- Sodium channel extracellular subunits can be stained without tissue fixation/permeabilisation

- Sodium channel clustering at nodes of Ranvier can me imaged during nerve straining

- Axonal strains as a function of applied tissue strain can be estimated by digital image correlation 


\title{
Strain partitioning between nerves and axons:
}

\section{estimating axonal strain using sodium channel staining in intact peripheral nerves}

\author{
Fabio Bianchi ${ }^{1}$, Ruby Sedgwick ${ }^{2}$, Hua Ye $^{1}$ and Mark S. Thompson ${ }^{1 *}$ \\ ${ }^{1}$ Institute of Biomedical Engineering, Dept. of Engineering Science, University of Oxford, \\ Oxford OX3 7DQ, UK \\ 2 Department of Engineering Science, University of Oxford OX1 3PJ, UK \\ E-mail: hua.ye at eng.ox.ac.uk
}

\footnotetext{
* Corresponding Author Prof. Mark S. Thompson, Institute of Biomedical Engineering, Botnar Research Centre, Headington, Oxford OX3 7LD, UK. Tel: +44(0)1865 737845
} 


\section{Abstract.}

\section{Background}

Peripheral nerves carry afferent and efferent signals between the central nervous system and the periphery of the body. When nerves are strained above physiological levels, conduction blocks occur, resulting in debilitating loss of motor and sensory function. Understanding the effects of strain on nerve function requires knowledge of the multi-scale mechanical behaviour of the tissue, and how this is transferred to the cellular environment.

New method

The aim of this work was to establish a technique to measure the partitioning of strain between tissue and axons in axially loaded peripheral nerves. This was achieved by staining extracellular domains of sodium channels clustered at nodes of Ranvier, without altering tissue mechanical properties by fixation or permeabilisation.

Results

Stained nerves were imaged by multi-photon microscopy during in-situ tensile straining, and digital image correlation was used to measure axonal strain with increasing tissue strain. Strain was partitioned between tissue and axon scales by an average factor of 0.55.

Comparisons with existing methods

This technique allows non-invasive probing of cell-level strain within the physiological tissue environment.

Conclusions

This technique can help understand the mechanisms behind the onset of conduction blocks in injured peripheral nerves, as well as to evaluate changes in multi-scale mechanical properties in diseased nerves.

Keywords: Peripheral nerve, uniaxial strain, nerve damage, strain partitioning, axon strain 


\section{Introduction}

The peripheral nervous system lacks ossified protective structures, exposing it to continuous mechanical deformation. Supraphysiological elongation of peripheral nerves causes injury, which can range from mild discomfort to complete transection [1]. At strains of 5-20\% functional deficits occur, which result in impaired sensory and motor function $[2,3]$.

The mechanism that leads to conduction deficits is not entirely understood. Peripheral nerves are mechanically modelled as concentric composites, made up of an outer sheath (consisting of the thick collagenous epineurium and outer perineurium), and a soft inner core, containing myelinated and unmyelinated axons, connected by a layer which allows relative sliding (mesoneurium) $[4,5,6]$. It has been hypothesised that excessive axonal elongation causes excessive separation of nodes of Ranvier, resulting in impaired signal conduction $[7,8]$. Clinically, nerve elongation occurs as a result of traumatic injury such as joint dislocation [9], or chronically due to compression syndromes such as carpal tunnel syndrome, where localised compression results in nerve stretch during wrist movement [10]. Understanding the mechanical properties of peripheral nerve is also of considerable importance in designing artificial nerve grafts and conduits. Tissue engineering approaches to nerve repair must

consider the biomechanical properties of the tissue, to design compatible replacements [11]. The partitioning of axial strain between tissue and axons is not understood, and could help explain altered functionality as a result of stretch. This has previously been investigated in fibrous tissues, with implications in tissue engineering designs [12]. A better understanding of strain transfer in peripheral nerve could impact the design on nerve grafts and artificial conduits, where biomechanical properties are increasingly recognised as fundamental design parameters [11].

Axonal damage thresholds have been estimated in central nervous system white matter 
by comparing morphological deformation and electrophysiological impairment [13], but do not provide information about the local axon strain. Multi-scale computational models of brain mechanics account for partitioning of strain between tissue and axon, but lack noninvasive experimental confirmation $[14,15]$. Fourier analysis of periodic Bands of Fontana that result from axon tortuosity has been used to estimate microstructural strains, but do not provide a reliable direct measurement of axonal strain [16].

Nodes of Ranvier, the periodically spaced gaps between myelinating Schwann cells, present an ideal intrinsic feature to measure axon strain. In previous work, contactinassociated proteins (CASPR) have been stained, and used as fiducial markers during spinal cord elongation [17]. However, CASPR staining requires paraformaldehyde fixation and surfactant permeabilisation, which significantly alter tissue mechanical properties [18, 19]. Sodium channels, which cluster at nodes of Ranvier, are formed of four transmembrane alpha subunits - and one transmembrane beta subunit - which contains an extracellular domain accessible without permeabilisation (figure 1) [20].

Digital image correlation (DIC) is a non-invasive technique which uses surface feature matching to calculate strains between images, and has been previously used to evaluate strain partitioning between meniscus tissue and chondrocytes [21] and within annulus fibrous cells $[22]$.

The aim of this study is to measure localised axonal strains during whole-nerve elongation to quantify axial strain partitioning in peripheral nerves, using multi-photon imaging of nerves without fixation or permeabilisation. This was achieved by staining of extracellular domains of sodium channel beta subunits in nodes of Ranvier by immunocytochemistry without fixing or permeabilising the nerve, and using DIC to estimate axonal strain during tissue elongation. The onset of Wallerian degeneration, which causes axonal breakdown in injured nerves, is delayed by freezing of excised nerves [23], which has been shown not to alter the mechanical 
properties of the tissue [24]. This technique can be further applied to studying alterations in axon mechanical properties in diseased nerves, as well as helping understand multi-scale strain partitioning for nerve tissue engineering.

\section{Materials and Methods}

\subsection{Sciatic Nerve Harvesting}

Sciatic nerves were harvested from 300-350 g (10-12 week old), male Sprague-Dawley rats, sacrificed by cervical dislocation for an unrelated study. Briefly, following skin removal, the sciatic nerves were exposed by dorsal incision of the gluteus muscle. Nerves were excised proximally close to the spinal cord, and distally at the tibial-peroneal branching. Nerve samples were stored in Phosphate Buffered Saline (PBS, Gibco, UK), immediately frozen at $-20^{\circ} \mathrm{C}$ until use. Freezing and thawing has been extensively used in nerve biomechancal experiments $[25,26,27]$, and shown not to alter specific mechanical properties of peripheral nerves [24] as well as those of other collagenous tissues [28], and lowering tissue temperature has been shown to delay the onset of Wallarian degeneration [23].

\subsection{Sodium Channel Staining}

Sciatic nerves were incubated at $4^{\circ} \mathrm{C}$ in $1: 50$ dilution of primary antibodies against extracellular sodium channel subunits I and IV (Alomone Labs, Israel) in PBS with 1\% bovine serum albumin (BSA, Sigma Aldrich, UK), for 48 hours on an orbital shaker. Samples were then washed in PBS with 1\% BSA five times, for five minutes each time, and incubated with AlexaFluor488 secondary antibody (ThermoFisher) for a further 48 hours at $4^{\circ} \mathrm{C}$ on an orbital shaker. Samples were then washed twice more in cold PBS, and mounted on glass coverslips or stretching machine for imaging. A control for non-specific staining was carried 

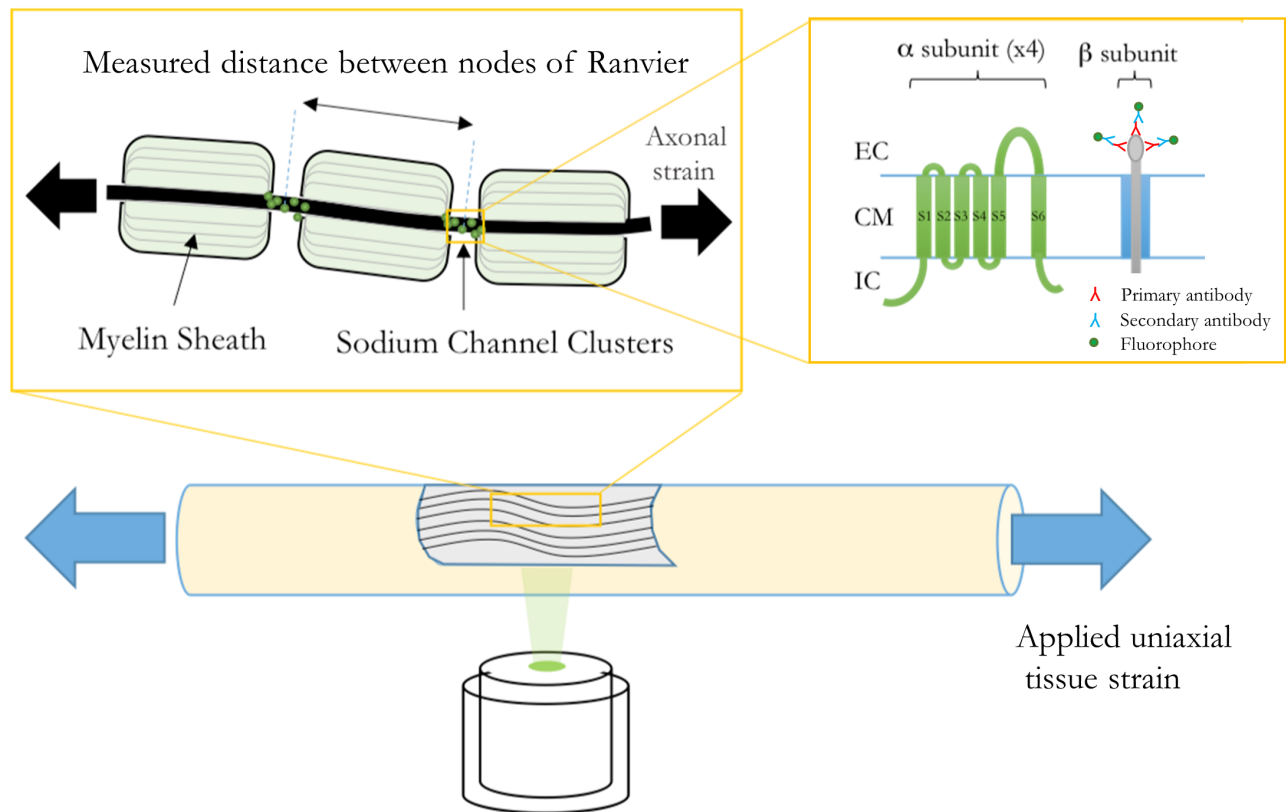

Multi-Photon Microscope

Figure 1: Experimental setup overview. Rat sciatic nerves were subject to displacement controlled tensile loading, and induced axonal strains were measured by digital image correlation of multi-photon images of stained sodium channels clustered at nodes of Ranvier. Inset shown sodium channel structure, with primary antibody targeted to extracellular $\beta$-subunit and secondary antibody (fluorophore) binding to primary antibody. $\mathrm{EC}=$ extracellular, $\mathrm{CM}=$ cell membrane, $\mathrm{IC}=$ intracellular

out by incubating nerve tissue with secondary fluorophore only, resulting in no fluorescent signal after washing.

\subsection{Nerve Stretching and Imaging}

To test the antibody protocol, stained nerves were stripped of the epineurium, and individual fibres teased using fine forceps. Fibres were imaged using a Zeiss LSM 708 inverted confocal microscope (100x oil immersion lens), simultaneously acquiring green fluorescence and transmitted light signals. Whole nerves were imaged during stretch using multi-photon microscopy (MPM), which allows imaging through thicker tissue sections. MPM images (20x lens) were taken using a Zeiss LSM 780 inverted microscope during graded nerve elongation, 


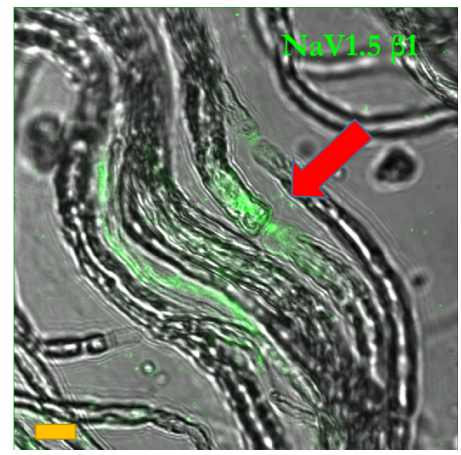

(a)

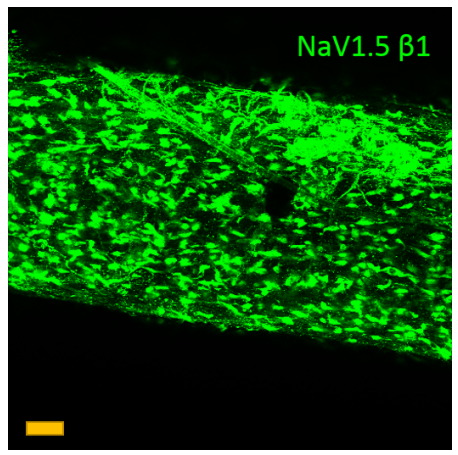

(b)

Figure 2: (a) Co-localisation of node of Ranvier morphology (red arrow, transmitted light image) and sodium channels (NaV1.5 $\beta$ subunit, green), imaged by confocal microscopy in excised rat sciatic nerves, stained without fixation or permeabilisation. Scale bar $=10 \mu \mathrm{m}$. (b) Maximum intensity projection of multi-photon imaging z-stack through nerve. Scale bar $=50 \mu \mathrm{m}$.

applied using a custom-built uniaxial stretching device with modified clamps directly gripping the nerves [29]. Nerves were stretched in 5\% grip-to-grip strain increments, from 0\% to $20 \%$, at $0.1 s^{-1}$ strain rate. This strain range starts below in vivo level of pre-strain, in order to construct a full plot for strain partitioning up to strains recognised as the threshold for conduction blocks $[2,3]$. Z-stack images with slice thickness of $4 \mu \mathrm{m}$ were taken at each value of applied strain, with laser power correction to account for nerve thickness. Nerves were kept hydrated by manual addition of PBS. Five nerves were prepared for testing, of which four are reported here. One sample (nerve 4) failed due to slipping at the clamp grip at $20 \%$ applied strain. Another sample was damaged during mounting.

\subsection{Digital Image Correlation}

Average axonal strain was estimated by DIC. Maximum intensity projections of z-stacks of stained nerves were used as patterns to calculate average strains. GOM Correlate (GOM GmbH, Germany) was used to calculate strain parallel to elongation direction between images from successive applied deformation levels. Only points which were successfully tracked at all 
levels of applied strain were used for analysis. DIC measurements correspond to an average axonal strain in the same direction as tissue loading. Compared to tracking individual axons [17], this method does not require manual tracking of individual axons (which would not be possible without further staining requiring fixation and permeabilisation) and returns a higher number of points used for correlation.

\subsection{Statistical Analysis}

Statistical analysis was carried out using PRISM (GraphPad, USA). Data was fitted by linear regression. Changes in measured axonal strain with applied nerve strain were tested for significance by one way paired ANOVA testing, with Tukeys multiple comparison between successive applied strain values. Results reported as significant if $\mathrm{p}<0.05$.

\section{Results and Discussion}

\subsection{Sodium Channels can be Stained Without Fixation and Permeabilisation}

Confocal images of stained teased fibres show co-localisation of sodium channel staining and nodes of Ranvier (figure 2a), confirming that the protocol used is suitable for wholenerve preparations without fixation or permeabilisation. Multi-photon z-stacks show staining of nodes of Ranvier within the thickness of the nerve, from which a maximum intensity projection can be used to obtain a pattern for DIC (figure 2b). Previously Singh et al. estimated white matter axonal strains during tissue deformation by following paranodal regions of nodes of Ranvier. In their experiments, spinal cords were stretched, and fixed overnight in the deformed position. Tissues were then sliced, permeabilised using Triton X-100 and stained for CASPR, found at paranodal regions of nodes of Ranvier, which were used as fiducial markers to estimate axonal strains [17]. Fixation of tissues cross-links cellular 

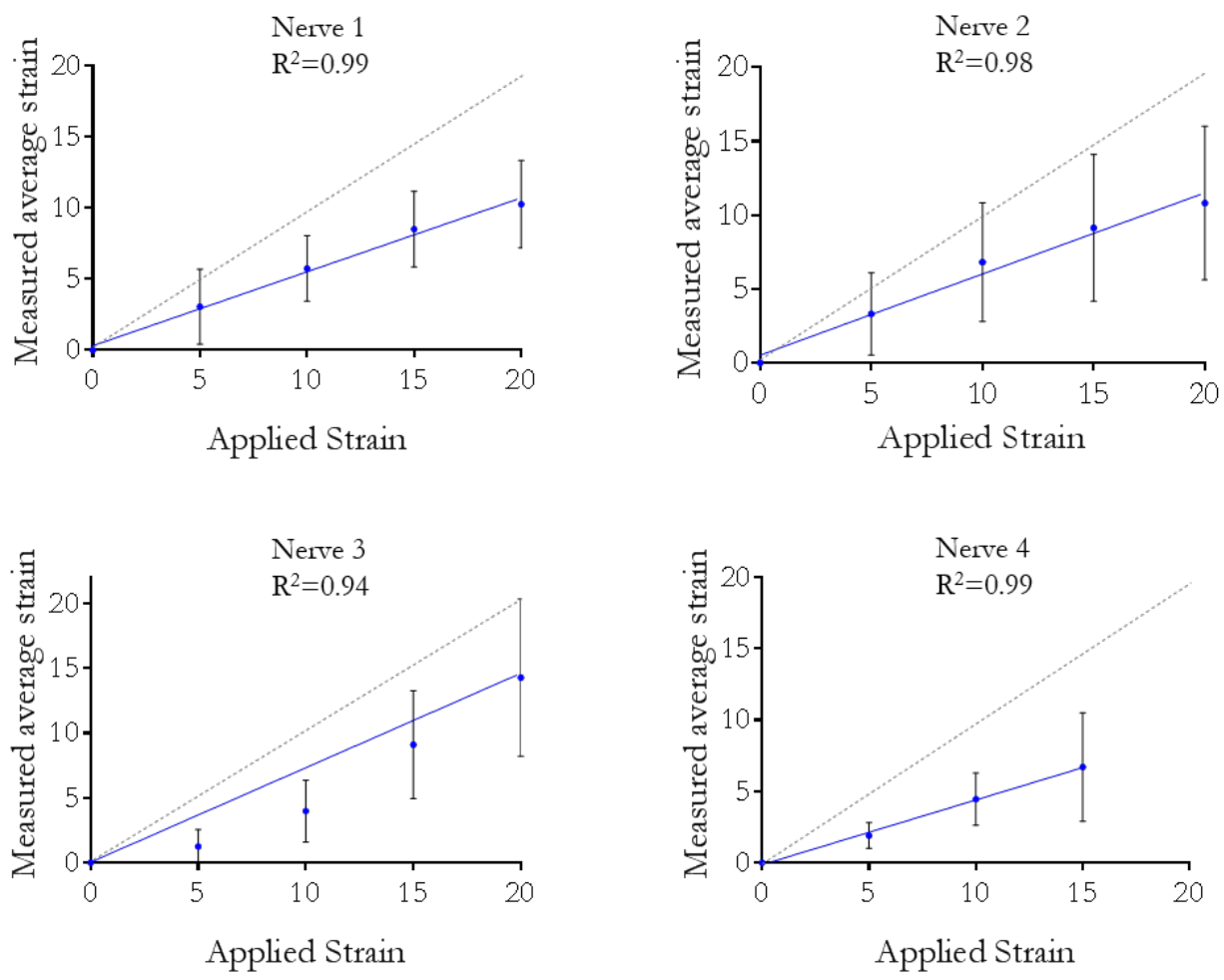

Figure 3: Measured axonal strain (\%) increases linearly with applied tissue strain (\%) for all four nerves tested. Points show average, errors bars show S.D. Blue lines are linear regression fits. Dotted lines show unity slope.

structure, irreversibly altering the mechanical properties of the tissue [18]. Furthermore, slicing alters tissue ultrastructure, possibly resulting in altered mechanical properties. In the method presented here, staining extracellular elements does not require permeabilisation of the cell membrane using surfactants, and freezing allows tissue preservation without fixation. Stretched nerves therefore closely resemble in vivo conditions.

\subsection{Measured Axonal Strain Increases Linearly with Tissue Strain}

DIC was used to estimate the average strain at the axon level, by averaging over 50 points for each applied strain level. For all four nerves tested, measured average axonal strain increased linearly with applied tissue strain (figure $3, \mathrm{R}^{2}=0.99,0.98 .0 .94$ and 0.99 ). In all four nerves, axonal strain increased significantly between each incremental level of applied strain $(\mathrm{p}<$ 
0.001 between successive strains for all nerves), with a small deviation from linear behaviour observed at higher strains for nerves one and two. Singh et al. report a similar nonlinearity in spinal cords [17], attributing this to axon disconnection during stretch. Bain and Meaney [13] have estimated by comparing morphological and electrophysiological changes in white matter, a threshold of approximately $20 \%$ tissue strain which causes axonal damage, in line with strain values where we see the greatest departure from linearity. The average slope from linear regression was $0.56 \pm 0.10$, representing the ratio between applied strain and measured strain. Strain transfer properties have been shown to vary between tissue types, depending on tissue architecture and isotropy [12]. In peripheral nerves, where axons are constrained within endoneurial collagen tubes surrounded by stiff perineurial and epineurial collagen strain may partition axially to avoid excessive axonal elongation. Peripheral nerve axons are known to be crimped, less stiff than surrounding perineurial and epineurial collagen, and connected to the outer sheath only by the thin mesoneurial layer that allows sliding $[6,5]$. When the nerve is strained, the stiffer collagenous tissue will limit the overall extension. The axial collagen layer also exhibits crimp visible under MPM second harmonic generation, which decreases with applied strain but does not completely disappear at $20 \%$ strain (data not shown). Axons within the nerve core will uncrimp, and slide within the stiffer outer sheath, resulting in lower axonal strain. Love et al. showed, using Fourier analysis of periodic bands of Fontana, that nerves retain some periodic axonal waviness at strains up to $20 \%$ [16]. This indicates that in our experiments axons will not be fully uncrimped at maximum applied strains (20\%). Due to the well-established differences in mechanical properties between nerve core and outer sheath $[24,5]$, these results provide further evidence of a protective mechanical role for outer sheath collagen. By allowing sliding of nerve core and outer sheath, the full load applied to the tissue is not transferred to the core, thereby protecting the softer axons from mechanical damage. In the outer sheath, collagen fibres are also crimped when unloaded. 
It has been shown, however, that collagen uncrimps significantly faster than axons [16, 30], again suggesting that the outer sheath will protect the underlying core during stretch by bearing load before axons are strained beyond repair. Schwann cells are known to have a significant role in providing resilience and compressive strength to axons [31]. Axially, spinal cord demyelination has been shown to lower tissue stiffness and ultimate tensile strength, suggesting a mechanical role for myelin, although demyelination methods may directly alter tissue mechanical properties [32]. Furthermore, nodes of Ranvier have been shown to elongate significantly during nerve stretch [33]. Because we use inter-nodal distance to estimate axonal strain, our results may underestimate axonal elongation.

\section{Conclusions}

Conduction deficits and loss of sensory and motor function are known to occur when peripheral nerves are stretched supraphysiologically. A multi-scale understanding of nerve mechanics is required to understand the damage processes. Currently, the mechanical relationship between tissue deformation and axonal strain is not well characterised. Current techniques are limited because sample preparation alters the mechanical properties of the tissue. Here, we show that average axonal strain can be estimated by multi-photon microscopy of whole nerves, stained for sodium channel extracellular subunit beta-1, without tissue fixation or permeabilisation. We show that axons strain increases linearly with applied tissue strain, with a slope of approximately 0.55 , indicating that axons only take up half the tissue deformation. Both axons and collagen exhibit crimp patterns that allow the nerve to withstand high strains without significantly loading the axonal fibres. This experimental approach can be used in the future to study strain partitioning between tissue and axons in models of degenerative diseases. The results presented here can be used to calibrate singlecell deformation models, aimed at eliciting the relationship between nerve stretch and neural 
function loss. Although these conclusions are limited by the small number of nerves tested,

the method presented should be of interest for understanding the biomechanical properties of peripheral nerve, and how they translate to impaired neural function.

\subsection{Acknowledgements}

This work was supported by the Rosetrees Trust (award M186-F1) and China Regenerative Medicine International Limited (CRMI) for materials, and EPSRC for F.B. funding through DTP award 1514540.

\section{References}

[1] Kimberly S Topp and Benjamin S Boyd. Structure and biomechanics of peripheral nerves: nerve responses to physical stresses and implications for physical therapist practice. Phys. Ther., 86(1):92109, January 2006.

[2] Shinro Takai, Hidefumi Dohno, Yoshinobu Watanabe, Nobuyuki Yoshino, Taku Ogura, and Yasusuke Hirasawa. In situ strain and stress of nerve conduction blocking in the brachial plexus. J. Orthop. Res., 20(6):1311-1314, November 2002.

[3] E J Wall, J B Massie, M K Kwan, B L Rydevik, R R Myers, and S R Garfin. Experimental stretch neuropathy. changes in nerve conduction under tension. J. Bone Joint Surg. Br., 74(1):126-129, January 1992.

[4] Rachel L Tillett, Andrew Afoke, Susan M Hall, Robert A Brown, and James B Phillips. Investigating mechanical behaviour at a core-sheath interface in peripheral nerve. J. Peripher. Nerv. Syst., 9(4):255262, December 2004.

[5] G A Georgeu, E T Walbeehm, R Tillett, A Afoke, R A Brown, and J B Phillips. Investigating the mechanical shear-plane between core and sheath elements of peripheral nerves. Cell Tissue Res., 320(2):229-234, May 2005.

[6] H Millesi, G Zöch, and R Reihsner. Mechanical properties of peripheral nerves. Clin. Orthop. Relat. Res., (314):76-83, May 1995.

[7] Franklin Rivera Bueno and Sameer B Shah. Implications of tensile loading for the tissue engineering of nerves. Tissue Eng. Part B Rev., 14(3):219-233, September 2008.

[8] Harumitsu Ichimura, Takashi Shiga, Ichiro Abe, Yuki Hara, Naoto Terui, Akihito Tsujino, and Naoyuki Ochiai. Distribution of sodium channels during nerve elongation in rat peripheral nerve. J. Orthop. Sci., 10(2):214-220, 2005.

[9] Christopher A Taylor, Diane Braza, J Bradford Rice, and Timothy Dillingham. The incidence of peripheral nerve injury in extremity trauma. Am. J. Phys. Med. Rehabil., 87(5):381, May 2008.

[10] Annina B Schmid, Jeremy D P Bland, Manzoor A Bhat, and David L H Bennett. The relationship of nerve fibre pathology to sensory function in entrapment neuropathy. Brain, 137(Pt 12):3186-3199, December 2014.

[11] Xiaosong Gu, Fei Ding, Yumin Yang, and Jie Liu. Construction of tissue engineered nerve grafts and their application in peripheral nerve regeneration. Prog. Neurobiol., 93(2):204-230, February 2011. 
[12] Woojin M Han, Su-Jin Heo, Tristan P Driscoll, Lachlan J Smith, Robert L Mauck, and Dawn M Elliott. Macro- to microscale strain transfer in fibrous tissues is heterogeneous and tissue-specific. Biophys. J., 105(3):807-817, August 2013.

[13] A C Bain and D F Meaney. Tissue-level thresholds for axonal damage in an experimental model of central nervous system white matter injury. J. Biomech. Eng., 122(6):615-622, December 2000.

[14] A Jerusalem, J A Garcia-Grajales, A Merchan-Perez, and J M Pena. A computational model coupling mechanics and electrophysiology in spinal cord injury. Biomech. Model. Mechanobiol., 13:883-896, 2014.

[15] K Ueno, J W Melvin, L Li, and J W Lighthall. Development of tissue level brain injury criteria by finite element analysis. J. Neurotrauma, 12(4):695-706, August 1995.

[16] James M Love, Ting-Hsien Chuang, Richard L Lieber, and Sameer B Shah. Nerve strain correlates with structural changes quantified by fourier analysis. Muscle Nerve, 48(3):433-435, September 2013.

[17] Sagar Singh, Assimina A Pelegri, and David I Shreiber. Estimating axonal strain and failure following white matter stretch using contactin-associated protein as a fiduciary marker. J. Biomech., 51:32-41, January 2017.

[18] Seong-Oh Kim, Joonhui Kim, Takaharu Okajima, and Nam-Joon Cho. Mechanical properties of paraformaldehyde-treated individual cells investigated by atomic force microscopy and scanning ion conductance microscopy. Nano Converg, 4(1):5, March 2017.

[19] J S Cartmell and M G Dunn. Effect of chemical treatments on tendon cellularity and mechanical properties. J. Biomed. Mater. Res., 49(1):134-140, January 2000.

[20] Sivakumar Namadurai, Nikitha R Yereddi, Fiona S Cusdin, Christopher L H Huang, Dimitri Y Chirgadze, and Antony P Jackson. A new look at sodium channel $\beta$ subunits. Open Biol., 5(1):140192, January 2015.

[21] Maureen L Upton, Christopher L Gilchrist, Farshid Guilak, and Lori A Setton. Transfer of macroscale tissue strain to microscale cell regions in the deformed meniscus. Biophys. J., 95(4):2116-2124, August 2008.

[22] Christopher L Gilchrist, Sietske W Witvoet-Braam, Farshid Guilak, and Lori A Setton. Measurement of intracellular strain on deformable substrates with texture correlation. J. Biomech., 40(4):786-794, 2007.

[23] T Sea, M L Ballinger, and G D Bittner. Cooling of peripheral myelinated axons retards wallerian degeneration. Exp. Neurol., 133(1):85-95, May 1995.

[24] Erik T Walbeehm, Andrew Afoke, Thijs de Wit, Fabian Holman, Steven E R Hovius, and Robert A Brown. Mechanical functioning of peripheral nerves: linkage with the "mushrooming" effect. Cell Tissue Res., 316(1):115-121, April 2004.

[25] Elisabetta Giannessi, Maria Rita Stornelli, and Pier Nicola Sergi. A unified approach to model peripheral nerves across different animal species. PeerJ, 5:e4005, November 2017.

[26] Alejandro Heredia, Chin Chu Bui, Ueli Suter, Peter Young, and Tilman E Schäffer. AFM combines functional and morphological analysis of peripheral myelinated and demyelinated nerve fibers. Neuroimage, 37(4):1218-1226, October 2007.

[27] Zhijun Ma, Shihao Hu, Juay Seng Tan, Christopher Myer, Nina M Njus, and Zhenhai Xia. In vitro and in vivo mechanical properties of human ulnar and median nerves. J. Biomed. Mater. Res. A, 101(9):2718-2725, September 2013.

[28] Gion Fessel, Kevin Frey, Andreas Schweizer, Maurizio Calcagni, Oliver Ullrich, and Jess G Snedeker. Suitability of thiel embalmed tendons for biomechanical investigation. Ann. Anat., 193(3):237-241, May 2011.

[29] Jack Hornsby, Donna M Daly, David Grundy, Fangzhou Cheng, Anne M Robertson, Paul N Watton, and Mark S Thompson. Quantitative multiphoton microscopy of murine urinary bladder morphology during in situ uniaxial loading. Acta Biomater., 64:59-66, December 2017.

[30] C Stolinski. Structure and composition of the outer connective tissue sheaths of peripheral nerve. $J$. Anat., 186 ( Pt 1):123-130, February 1995. 
[31] Gonzalo Rosso, Ivan Liashkovich, Burkhard Gess, Peter Young, Alejandra Kun, and Victor Shahin. Unravelling crucial biomechanical resilience of myelinated peripheral nerve fibres provided by the schwann cell basal lamina and PMP22. Sci. Rep., 4:7286, December 2014.

[32] David I Shreiber, Hailing Hao, and Ragi A I Elias. Probing the influence of myelin and glia on the tensile properties of the spinal cord. Biomech. Model. Mechanobiol., 8(4):311-321, August 2009.

[33] K Ikeda, K Tomita, and S Tanaka. Experimental study of peripheral nerve injury during gradual limb elongation. Hand Surg., 5(1):41-47, July 2000. 